\title{
Gender-Specific Aspects in the Clinical Presentation of Cardiovascular Disease
}

\author{
Chiara Leuzzi, Raffaella Marzullo, \\ Emma Tarabini Castellani and Maria Grazia Modena* \\ Department of Cardiovascular Disease, Women's Clinic \\ University of Modena and Reggio Emilia, \\ Italy
}

\section{Introduction}

In the industrialized countries, the cardiovascular disease (CVD) is the leading cause of mortality and morbidity in women after of 50 years. Genetic, hormonal and metabolic influences are involved in gender differences, including the epidemiology, symptoms, diagnosis, progression, prognosis and management of these pathologies.

Recent advances in the field of cardiovascular medicine have not led to significant drops in case fatality rates for women, compared to the dramatic reductions achieved for men. Such gender-specific difference in cardiovascular disease mortality are probably related to a knowledge gap about CVD in women. Thus, much of the evidence supporting contemporary recommendations for testing, prevention, and treatment of CVD in women is extrapolated from studies conducted predominantly on middle-aged men. For example pharmacological therapy is hampered by defective evidence.

Only recently, significant sex-related differences in prevalence, presentation, management and outcomes of CVD, have been evaluated and discovered.

The ability of knowing and recognizing gender differences in CVD may facilitate a rapid identification of cardiac signs and symptoms of warning and may avoid significant delays in diagnosis and treatment in women. This compendium will briefly summarize genderrelated differences in several manifestations of CVD, with a special focus also on arrhythmias and heart failure.

\section{Risk factors}

Traditionally, guidelines classify women as being at high, intermediate or low risk on risk profile, based on Framingham risk scores. Despite major traditional risk factors are the same in both sexes, gender-specific differences are noted, and these differences are related to different outcome. For this reason, a new approach in evaluation of cardiovascular risk in

${ }^{*}$ Corresponding Author 
women considers a multifactorial model that includes a complex interaction between sex hormones and traditional risk factors.

\subsection{Traditional risk factors}

There is also substantial gender-related differences in the prevalence and outcome in traditional risk factors. For example, overall rates of hypertension and smoking are higher in men, but their presence is associated with a worse outcome in women. The prevalence of hypertension is lower in pre-menopausal women than men, whereas in post-menopausal women it is higher than in men. In fact about $50 \%$ of post-menopausal women experience of moderate to severe hypertension or take antihypertensive therapy.

Several studies have clearly demonstrated a strong relationship between level of blood pressure and risk for cardiovascular events. In this setting, hypertension is one of the most risk factors for stroke, myocardial infarction, heart failure, aortic disease and chronic renal failure. Mechanisms responsible for the increase in blood pressure in post-menopausal women are complex and multifactorial, including loss of estrogen, oxidative stress, endothelial dysfunction, modification in renin-angiotensin system and sympathetic activation.

Hypertension may appears as an isolated disease, more typical of elderly women, or as part of the metabolic syndromes (MS), more frequently in early postmenopausal women. MS is a constellation of interrelated risk factors that promote the development of CVD. The presence of MS worsens the severity of hypertension and reduces the response to treatment. Than, MS is considered a unfavourable prognostic factor in hypertension post-menopausal women. On the other hand hypertension tends to associate with other metabolic risk factors and about one-half patients with essential hypertension are insulin resistant. In addition women with MS have chronic subclinical inflammation and systemic endothelial dysfunction. Endothelial dysfunction is common after the menopause and its detection may precede overt disease such as hypertension and diabetes.

Data derived from population studies demonstrate that total cholesterol measurements are higher in men until the fifth decade of life but, beyond this age, women have greater values. Examining the lipoprotein subclassis, women have less LDL particles than men and have about two-fold higher concentration of HDL particles then men. Particularly, HDL cholesterol inversely correlates with coronary artery disease (CAD) in young men and in women of all ages. Women typically experience a relatively mild decline in HDL cholesterol at the time of menopause.

Hypertriglyceridemia is also a more potent independent risk factor for CAD in women as compared with men. In fact, in presence of hypertriglyceridemia, the risk of CVD is twice in women.

Another strong risk factors in women is diabetes. Diabetic women have significantly higher cardiovascular mortality when compared with diabetic men, because diabetes eliminates the 'female advantage' of a lower CAD prevalence and outcome risk that exists for the female in general population. Furthermore diabetes is an independent predictor of 'atypical' presentation of acute myocardial infarction in women. 
At the onset of diabetes-related cardiovascular complications, women have higher out-ofhospital mortality than men, and those who reach hospital are more likely to die from an initial cardiac event and are also at high risk of post-event complications.

\subsection{Gender-specific risk factors}

Other factors are unique to the female: menopause, which affects especially if early and hypothalamic-hypoestrogenism occurring in fertile women.

Menopause is a physiological condition associated with endothelial dysfunction, due to lack of estrogens. Than, the deficiency of female gonadal hormones may represent a major risk factor for menopausal hypertension, due to related modifications of blood vessel structure and the elicit response to a vasoactive substances.

Furthermore, dysfunction of the endotelium causes reduction or abolition of vasoprotective factors, inducing a proinflammatory, proliferative and procoagulatory milieu. These changes favour the development of cardiovascular risk factors, as hypertension.

Gender-specific opportunities for identifying women's risk (e.g., prior preeclampsia) also deserve further exploration.

Preeclampsia is a disorder of pregnancy diagnosed by gestational hypertension and proteinuria. Abnormal placentation resulting in preeclampsia and intrauterine growth restriction is a major cause of both maternal and perinatal morbidity and mortality.

Prior preeclampsia is associated with increased risk of CVD, including myocardial infarction, ischemic heart disease, stroke and endstage renal disease. Particularly, the increased risk for future vascular disease is more pronounced in women with early-onset preclampsia. Although the symptoms of preeclampsia resolve over a number of weeks after delivery, maternal vascular dysfunction may persist for years.

Endothelial dysfunction, however, is considered a central component of the pathophysiology of preeclampsia and known to contribute to the pathogenesis of hypertension and cardiovascular sequelae. Several factors contribute to the endothelial dysfunction in the post-partum state. Abnormal placenta, for example, release antiangiogenic factors, harmful to the vascular endothelium. Often, women with a history of peeclampsia or intrauterine growth restriction have high cholesterol levels, high blood pressure and insulin resistance.

Frequently, preeclamptic women are obese, and obesity associated with insulin resistance, may reduce endothelial dependent blood flow response.

Behavioral factors also, such as chronic stress, lack of social support, and family demands, as well as biological processes, including genetics, may contribute to the development of CVD in this setting.

Similarly, women with a history of polycystic ovary syndrome have a increased risk of CVD and have a greater frequency of multiple risk factors including central obesity, insulin resistance, and a greater prevalence of the metabolic syndrome and diabetes. 


\subsection{Inflammatory risk factors}

Beside the traditional and female specific risk factors, novel risk markers such as inflammatory markers are being studied.

Women are at increased risk of inflammatory and autoimmune disease. The risk of mortality and morbidity from CVD is very high in autoimmune diseases, as systemic lupus erythematosus or rheumatoid arthritis. Various possible mechanisms have been proposed to explain the excess rate of cardiovascular mortality in patients with autoimmune disease.

A combination of traditional (dyslipidemia, hypertension, diabetes, and smoking) and nontraditional risk factors, including high inflammation, antiphospholipid antibodies and lipid oxidation, contribute to CVD in autoimmune diseases. Inflammation is a key component in the development of atherosclerosis in this setting. In fact, inflammation leads to the activation of endothelial cells, which, through an increase in the expression of leukocyte adhesion molecules, promotes a pro-atherosclerotic environment. Expression of proinflammatory cytokines and inflammatory mediators influences all stages of atherosclerosis development, from early atheroma formation to thrombus development responsible for events such as myocardial infarction. Proinflammatory cytokines may promote both traditional (e.g., dyslipidemia, insulin resistance) and nontraditional (e.g., oxidative stress) systemic cardiovascular risk factors.

Than in these patients, is commonly found a presence of endothelial dysfunction, a loss of arterial compliance and dysfunction in the microvascolature, resulting in myocardial flow heterogeneity.

Others factors contribute to poor prognosis: undertreatment of cardiovascular comorbidity may contribute to increased cardiovascular mortality in these patients. However, some drugs, largely used in this setting, may worsen cardiovascular profile: e.g. corticosteroids promote hypertension, dyslipidemia, and diabetes.

Then, novel risk stratification, including inflammatory markers and reproductive hormones, is developing to assess global cardiovascular risk in women.

\section{Coronary heart disease}

Coronary heart disease (CHD) is the most common cause of death amongst women, who experience more complications after acute myocardial infarction (AMI) than men.

It has been demonstrated that the epidemiology, the clinical manifestation and the progression of CHD are different in both sexes. The women developed CHD about 10-20 years later than men, in part by the influence of hormones and in part by the genetic sex.

Particularly, at the time of first experience of AMI, women are more likely to have diabetes mellitus or heart failure (HF) than men.

In addition, the prevalence of obstructive coronary disease is particularly low in premenopausal women, whilst increases dramatically for a woman after age 50.

The most common initial presentation of CHD is a AMI or sudden cardiac death and up to half of all women presenting with an acute myocardial infarction report no prior chest pain 
symptoms. There would appear to be an interaction effect of symptom presentation with age, in that older women often present in a similar way to men.

Several studies have indicated that women have "atypical" symptoms such as back pain, dyspnea, indigestion, nausea/vomiting and weakness. Frequently women reported pain in the jaw and neck and describe their symptoms as more anguished and frightening (emotional component) compared with men. Furthermore, prodromal symptoms are described up to 1 month before the onset of AMI such unusual fatigue (70.7\%), sleep disturbance $(47.8 \%)$ and shortness of breath (42.1\%). The atypical presentation may explain the rate of under-diagnosed AMI, the under treatment of acute coronary syndromes and the worse outcomes characterized by increased hospital morbidity, higher mortality and fewer evidence-based therapies in women.

In the postmenopausal women, the plaque rupture is the main mechanism of acute coronary syndromes like as in the men. The higher mortality noted for younger women when compared with age-matched men is due the higher frequency of plaque erosion (Fig. 1). In an autopsy series, women also had a greater frequency of distal microvascular embolization in the setting of a fatal epicardial thrombosis when compared to men, independently of the type of thrombus or presence of necrosis. AMI and sudden death in women can occur also in spontaneous coronary artery dissection. This event is more frequent in the peri-partum period and can involve all coronary tree, but more frequently affects the left anterior descending artery. The dissection can involve every coronary, but in women frequently involves the left anterior descending coronary artery, whereas in man the right coronary artery is more frequently involved.

Many as $50 \%$ of patients undergoing coronary angiography for typical or atypical chest pain do not have obstructive CAD. An alternative mechanism of pain in women may be a coronary microvascular dysfunction, known as syndrome $X$. Most of these patients have an 'abnormal' exercise stress test, myocardial perfusion defects on gated Single-photon emission computed tomography or stress-induced wall motion abnormalities on echocardiography, but normal coronary angiography.

However, differentiation between these mechanisms of chest pain is important, because 'noncardiac' chest pain is not associated with cardiovascular sequelae and may require further medical evaluation and treatment. By contrast, syndrome $X$, which is thought to be caused by microvascular dysfunction, is associated with inducible metabolic ischaemia and can be treated by improving microvascular vasomotor tone with oral L-arginine, a precursor to vascular nitric oxide, and oestrogen.

Despite the absence of CAD and low risk for adverse cardiac events, a majority of those patients continue to have symptoms that contribute to a poor quality of life and consumption of large amounts of health care resources because of repeated evaluations and hospitalizations. Recently, Han et al. studied patients with obstructive CAD who underwent simultaneous intravascular ultrasound and coronary reactivity assessment and demonstrated that men have a greater atheroma burden and more diffuse epicardial endothelial dysfunction while women have more disease of the microcirculation. The coronary micro-vascular dysfunction, the smaller coronary artery lumens, the less collateral circulation than men and more prominent positive remodeling support the higher rate of 
angina, and acute coronary syndromes in the absence of obstructive CAD particularly during exertion or stress.

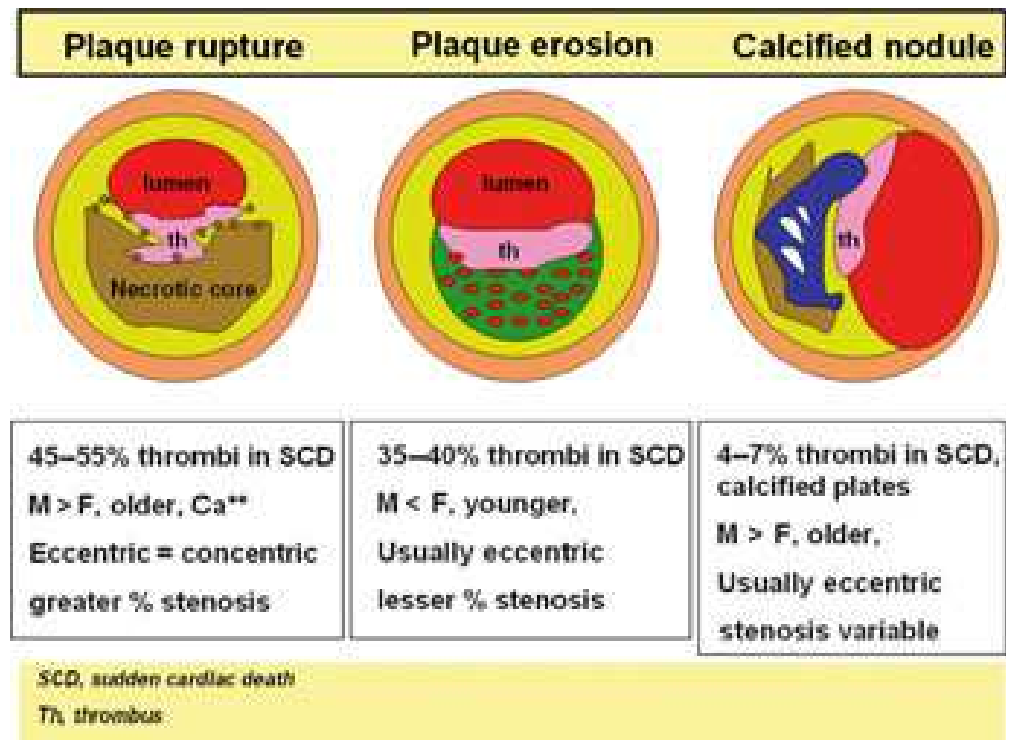

SCD, sudden cardiac death; Th, thrombus

Fig. 1. Gender differences in plaque morphologic features in an autoptic series of patients who died for sudden cardiac death, modified from reference 13.

\section{Heart failure}

The lifetime risk of developing HF is about $20 \%$ for both men and women. There are differences between men and women in clinical presentation, aetiology, treatment and outcome in HF, and these differences lead to different outcome.

The women are older than men and present more frequent hypertension and diabetes.

Diabetes mellitus is one of the strongest additional risk factors for the development of HF in women with CAD.

The systolic function is usually better preserved and the prevalence of ischemic etiology is lower respect to hypertension and valvular diseases. Another cause of HF includes cardiac toxicity from chemotherapeutic agents used to treat breast malignancy.

Mullens et al. show that the survival rate in women with non-ischemic cardiomyopathy was better than men, irrespective of baseline characteristics, while there was no advantage in presence of ischemic cause. The reason of different outcome remains unclear but might in part be related to sex differences in etiology.

The diagnosis of HF is a clinical diagnosis based on a constellation of symptoms and signs. Women with impaired systolic left ventricular function are more likely than men to have dependent edema, jugular venous distension, and an S3 gallop. 
Furthermore, normal brain natriuretic peptide value, a biomarker used to identify patients with symptoms of $\mathrm{HF}$, are higher in women versus men.

Current guidelines for HF therapy also are not sex specific due to under-representation of women and lack of sex-specific, prospective, randomized clinical trials. Indeed, women receive less life-prolonging treatment (ACE-I, beta-blockers and spironolactone) than men, in the presence of normal left ventricular function, while there no difference if etiology of $\mathrm{HF}$ is CAD.

Cardiac resynchronization therapy, an important therapy for HF, is beneficial for both women and men. Data suggest CRT is preferable to medical therapy alone in women for the combined end point of total mortality and hospital stay for major cardiovascular events.

A peculiar type of left ventricular dysfunction and HF typical in women is Takotsubo cardiomyopathy. This disease is typically observed in post-menopausal women and the highest frequency of occurrence is between the seventh and eighth decade of life.

The reason for the much more common occurrence in postmenopausal women is unclear.

It is characterized by a left ventricular dysfunction, electrocardiographic changes like an acute myocardial infarction and release of cardiac biomarkers, in the absence of obstructive coronary disease. The emotionally or physical stress are usually the triggers. The catecholamine-mediated cardio-toxicity, multi-vessels coronary vasospasm and abnormalities in coronary micro-vascular function have been postulated as pathophysiologic mechanisms.

The left ventricular dysfunction is reversible within weeks, despite a dramatic clinical presentation (similar to acute myocardial infarction but in the absence of obstructive coronary disease) and substantial risk of complications in the acute setting.

Another cause of left ventricular dysfunction and HF typical in women is peripartum cardiomyopathy (PPCM). This disease develops in the last month of pregnancy or within 5 months post-partum with no pre-existing cardiac disease or identifiable cause. The incidence is very low $(<1 \%)$ and varies on the basis of the population studied. Risk factors include advanced maternal age, African descent, twin pregnancy, usage of tocolytics, and poverty.

The etiology remains unknown, but potential causes include abnormal immune response to pregnancy, increased myocyte apoptosis, genetic predisposition. Only $20 \%$ may worsen up to the death or transplantation, while one-half of PPCM patients recover normal systolic function within 6 months.

\section{Arrhythmias}

It is been demonstrated that women had a higher resting heart rate than did men ( 3 to 5 beats faster for minute). These finding may be explained by differences in exercise tolerance, autonomic modulation and intrinsic properties of the sinus node, influencing in part by hormonal influences. Burke et al. reported an higher average heart rate during the follicular or luteal phases of the menstrual cycle, although the response to double autonomic blockade was identical regardless of phase. 
Several authors demonstrated differences in QT interval between men and women. Women have a longer corrected QT interval and the difference becomes more pronounced at lower heart rates. Rautaharju et al. reported that this difference was due to a drop in the corrected QT that occurred in males after puberty (when androgen levels are highest). Then, the interval in men gradually increased with age until 50 years, at which point it paralleled that of women. The actions of hormonal influences in QT differences was confirmed in families with genotipically characterized long QT syndrome. In addition, the torsades de pointes correlated to both congenital and acquired long QT Syndrome was more frequent in women.

There are many differences in incidence, prevalence, presentation and clinical course of many arrhythmias. Inappropriate sinus tachycardia is more common in women. Rodigruez et al. reported in patients undergoing invasive electrophysiologic testing for various tachycardias that the atrio-ventricular node reentrant tachycardia (AVNRT) was twice times more common in women, whereas atrial tachycardia affected both sexes equally. In addition, the atrio-ventricular reentrant (circus-movement) tachycardia (AVRT), atrial fibrillation (AF), and ventricular fibrillation (VF) occurred more often in men. These trends for supra-ventricular tachycardias (SVTs), AF, VF, were confirmed in the Framingham cohort. Myerburg and associates found that the inducing SVT during electrophysiologic testing was greatest at the onset of menses or during the premenstrual phase. Rossano et al. confirmed that the SVT prone state is hindered by estrogen and facilitated by progesterone.

The SVTs new- onset or exacerbation are most common arrhythmia during pregnancy and the postpartum period in absence of structural heart disease. The mechanisms for increase in this situation may be related to progesteronerich gravid state, increased intravascular volume and autonomic tone.

The Atrial Fibrillation (AF) is the most prevalent in men, although its incidence increases with age both in men and women. Women with AF are more symptomatic, older and have lower quality of life and more co-morbidities than men. Also they present more likely a higher heart rate, longer episodes and increase incidence of embolic strokes compared to men. Data from the Euro Heart Survey on Atrial Fibrillation demonstrated that women are usually treated less aggressively, with fewer cardioversions and catheter ablations. In this study, albeit both genders received anticoagulation therapy, women experienced a significantly higher rate of stroke and major bleeding events.

\section{References}

[1] Leuzzi C, Sangiorgi GM and Modena MG (2010) Gender-specific aspects in the clinical presentation of cardiovascular disease Fundam Clin Pharmacol. N. 24(6):711-7

[2] Athyros VG, Ganotakis E, Kolovou GD et al (2011). Assessing The Treatment Effect in Metabolic Syndrome without Perceptible Diabetes (ATTEMPT): A ProspectiveRandomized Study in Middle Aged Men and Women. Curr Vasc Pharmacol Apr 11. [Epub ahead of print].

[3] Leuzzi C, Modena MG (2011). Hypertension in postmenopausal women: pathophysiology and treatment. High Blood Press Cardiovasc Prev. N. 18;13-8.

[4] Shaw LJ, Bugiardini R, and Bairey Merz CN (2009) Women and Ischemic Heart Disease: Evolving Knowledge J. Am. Coll. Cardiol. N: 54;1561-1575

[5] Bairey Merz CN et al.(2006) Insights from the NHLBI-Sponsored Women's Ischemia Syndrome Evaluation (WISE) Study: Part II: gender differences in presentation, 
diagnosis, and outcome with regard to gender-based pathophysiology of atherosclerosis and macrovascular and microvascular coronary disease. J. Am. Coll. Cardiol. N:47 S21-S29

[6] Vitale C, Miceli M, Rosano GM.(2007) Gender-specific characteristics of atherosclerosis in menopausal women: risk factors, clinical course and strategies for prevention. Climacteric. 2007 N.10; 16-20

[7] Association A.H.. Heart Disease and Stroke Statistics: 2004 Update. available at: http:/ / americanheart.org/downloadable/heart/1072969766940HSStats2004Updat e.pdf

[8] Yinon Y, Kingdom JCP, Odutayo A (2010). Vascular Risk Vascular Dysfunction in Women With a History of Preeclampsia and Intrauterine Growth Restriction : Insights Into Future. Circulation N. 122, 1846-1853

[9] Bairey Merz N et al.(2004) Women's Ischemic Syndrome Evaluation: current status and future research directions: report of the National Heart, Lung and Blood Institute workshop: October 2-4, 2002: executive summary. Circulation N:109, 805-807

[10] Bugiardini R, Estrada JL, Nikus K et al. (2010) Gender bias in acute coronary sindromes. Curr Vasc Pharmacol. N.8;276-84

[11] Vaccarino $\mathrm{V}$ et al.(1999) Sex-based differences in early mortality after myocardial infarction. National Registry of Myocardial Infarction 2 Participants. N. Engl. J. Med. N:341 217-225

[12] Nabel EG et al.(2002) Women's Ischemic Syndrome Evaluation: current status and future research directions: report of the National Heart, Lung and Blood Institute workshop: October 2-4, 2002: Section 3: diagnosis and treatment of acute cardiac ischemia: gender issues. Circulation N: 109 e50-e52. 25

[13] Kolodgie FD et al. (2004) Pathologic assessment of the vulnerable human coronary plaque. Heart N: 90 1385-1391. 26

[14] Farb A et al. (2003) Pathological mechanisms of fatal late coronary stent thrombosis in humans. Circulation N:108, 1701-1706

[15] Shaver PJ, Carrig TF and Baker WP (1978)Postpartum coronary artery dissection. Br. Heart J. N: 40 83-86

[16] Basso C, Morgagni G.L., Thiene G.(1996) Spontaneous coronary artery dissection: a neglected cause of acute myocardial ischaemia and sudden death. Heart N: 75, 451454

[17] Han SH et al.(2008)Sex differences in atheroma burden and endothelial function in patients with early coronary atherosclerosis. Eur Heart J N: 29, 1359-69

[18] Lloyd-Jones DM.(2001) The risk of congestive heart failure: sobering lessons from the Framingham Heart Study. Curr. Cardiol. Rep. N:3, 184-190

[19] O'Meara E et al.(2007) Sex differences in clinical characteristics and prognosis in a broad spectrum of patients with heart failure: results of the Candesartan in Heart failure: Assessment of Reduction in Mortality and morbidity (CHARM) program. Circulation N: 115 3111- 3120

[20] Hsich EM and Pina IL. (2009) Heart failure in women: a need for prospective data. J. Am. Coll. Cardiol. N: 54 491-498

[21] Mullens W et al. (2008) Gender differences in patients admitted with advanced decompensated heart failure. Am. J. Cardiol. N:102, 454-458

[22] Komajda M et al. (2003) The EuroHeart Failure Survey programme - a survey on the quality of care among patients with heart failure in Europe. Part 2: treatment. Eur.Heart J. N:24, 464-474 
[23] Koeth O et al.(2008) Clinical, angiographic and cardiovascular magnetic resonance findings in consecutive patients with Takotsubo cardiomyopathy. Clin. Res. Cardiol. N:97, 623-627

[24] Gianni M et al.(2006) Apical ballooning syndrome or takotsubo cardiomyopathy: a systematic review. Eur. Heart J. N: 27, 1523-1529

[25] Hsich EM and Piña IL. (2009). Heart Failure in Women: A Need for Prospective Data. J Am Coll Cardiol N.54; 491-498

[26] Liu $\mathrm{K}$ et al. (1989) Ethnic differences in blood pressure, pulse rate, and related characteristics in young adults. The CARDIA study. Hypertension N:14:218-26

[27] Linde C.(2000) Women and arrhythmias. Pacing Clin Electrophysiol N: 23:1550-60

[28] Kadish AH. (1995) The effects of gender on cardiac electrophysiology and arrhythmias. In: Zipes DP, Jalife J, editors. Cardiac electrophysiology: from cell to bedside. 2nd ed. Philadelphia: WB Saunders; p. 1268-75

[29] Huikuri HV et al. (1996) Sex-related differences in autonomic modulation of heart rate in middle-aged subjects. Circulation N:94,122-5

[30] Burke JH et al.(1997) Gender-specific differences in the QT interval and the effect of autonomic tone and menstrual cycle in healthy adults. Am J Cardiol N:79,178-81

[31] Stramba-Badiale $M$ et al.(1997) Gender and the relationship between ventricular repolarization and cardiac cycle length during 24-h Holter recordings. Eur Heart J $\mathrm{N}: 18: 1000-6$

[32] Lehmann MH et al. (1997) Age-gender influence on the rate-corrected QT interval and the QT-heart rate relation in families with genotypically characterized long QT syndrome. J Am Coll Cardiol N:29, 93-9

[33] Locati EH et al.(1998) Age- and sex-related differences in clinical manifestations in patients with congenital long-QT syndrome: findings from the International LQTS Registry. Circulation N: 97, 2237-44

[34] Yarnoz MJ and Curtis AB.(2008) More reasons why men and women are not the same (gender differences in electrophysiology and arrhythmias). Am. J. Cardiol. N: 101 1291-1296

[35] Rollo P et al.(2001) Gender and Cardiac Arrhythmias Tex Heart Inst J 28, 265-75

[36] Lee RJ, Shinbane JS.(1997) Inappropriate sinus tachycardia. Diagnosis and treatment. Cardiol Clin N:15,599-605

[37] Morillo CA, Klein GJ, Thakur RK, Li H, Zardini M, Yee R.(1994) Mechanism of 'inappropriate' sinus tachycardia. Role of sympathovagal balance. Circulation $\mathrm{N}: 90,873-7$

[38] Rodriguez LM et al. (1992) Age at onset and gender of patients with different types of supraventricular tachycardias.Am J Cardiol N:70:1213-5

[39] Benjamin EJ et al.(1994) Independent risk factors for atrial fibrillation in a populationbased cohort. The Framingham Heart Study. JAMA N:271,840-4

[40] Myerburg RJ et al. (1999) Cycling of inducibility of paroxysmal supraventricular tachycardia in women and its implications for timing of electrophysiologic procedures. Am J Cardiol N:83:1049-54

[41] Rosano GM et al.(1996) Cyclical variation in paroxysmal supraventricular tachycardia in women. Lancet $\mathrm{N}: 347,786-788$

[42] Yarnoz MJ, Curtis AB.(2008) More reasons why men and women are not the same (gender differences in electrophysiology and arrhythmias. Am. J. Cardiol. N:101, 1291-1296

[43] Dagres N et al. (2007) Gender-related differences in presentation, treatment, and outcome of patients with atrial fibrillation in Europe: a report from the Euro Heart Survey on Atrial Fibrillation. J Am Coll Cardiol N: 49, 572-577 


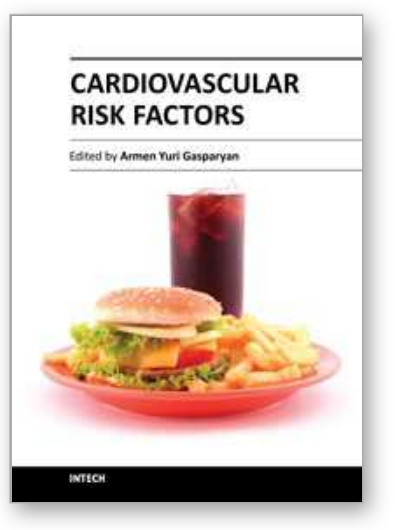

\author{
Cardiovascular Risk Factors \\ Edited by Prof. Armen Gasparyan
}

ISBN 978-953-51-0240-3

Hard cover, 498 pages

Publisher InTech

Published online 14, March, 2012

Published in print edition March, 2012

Cardiovascular risk factors contribute to the development of cardiovascular disease from early life. It is thus crucial to implement preventive strategies addressing the burden of cardiovascular disease as early as possible. A multidisciplinary approach to the risk estimation and prevention of vascular events should be adopted at each level of health care, starting from the setting of perinatology. Recent decades have been marked with major advances in this field, with the emergence of a variety of new inflammatory and immunemediated markers of heightened cardiovascular risk in particular. The current book reflects some of the emerging concepts in cardiovascular pathophysiology and the shifting paradigm of cardiovascular risk estimation. It comprehensively covers primary and secondary preventive measures targeted at different age and gender groups. Attention is paid to inflammatory and metabolic markers of vascular damage and to the assessment of vascular function by noninvasive standardized ultrasound techniques. This is a must-read book for all health professionals and researchers tackling the issue of cardiovascular burden at individual and community level. It can also serve as a didactic source for postgraduate medical students.

\title{
How to reference
}

In order to correctly reference this scholarly work, feel free to copy and paste the following:

Chiara Leuzzi, Raffaella Marzullo, Emma Tarabini Castellani and Maria Grazia Modena (2012). GenderSpecific Aspects in the Clinical Presentation of Cardiovascular Disease, Cardiovascular Risk Factors, Prof. Armen Gasparyan (Ed.), ISBN: 978-953-51-0240-3, InTech, Available from:

http://www.intechopen.com/books/cardiovascular-risk-factors/gender-specific-aspects-in-the-clinicalpresentation-of-cardiovascular-disease

\section{INTECH}

open science | open minds

\section{InTech Europe}

University Campus STeP Ri

Slavka Krautzeka 83/A

51000 Rijeka, Croatia

Phone: +385 (51) 770447

Fax: +385 (51) 686166

www.intechopen.com

\section{InTech China}

Unit 405, Office Block, Hotel Equatorial Shanghai

No.65, Yan An Road (West), Shanghai, 200040, China

中国上海市延安西路65号上海国际贵都大饭店办公楼405单元

Phone: +86-21-62489820

Fax: +86-21-62489821 
(C) 2012 The Author(s). Licensee IntechOpen. This is an open access article distributed under the terms of the Creative Commons Attribution 3.0 License, which permits unrestricted use, distribution, and reproduction in any medium, provided the original work is properly cited. 